


\title{
MONITORAMENTO ELETRÔNICO DAS RESPOSTAS COMPORTAMENTAIS DE VACAS EM LACTAÇÃO ALOJADAS EM FREESTALL CLIMATIZADO*
}

\author{
ELECTRONIC MONITORING ON BEHAVIORAL PATTERNS OF \\ DAIRY COWS IN A COOLING FREESTALL
}

\author{
SORAIA V. MATARAZZO' \\ IRAN J. O. DA SILVA2 \\ MAURICIO PERISSINOTTOO \\ SERGIO A. DE FERNANDES ${ }^{4}$ \\ DANIELLA J. DE MOURA ${ }^{5}$ \\ IRINEU ARCARO JÚNIOR ${ }^{6}$ \\ JULIANA R. P. ARCARO ${ }^{7}$
}

\section{RESUMO}

O trabalho teve como objetivo empregar o monitoramento eletrônico para avaliar as respostas comportamentais de vacas em lactação alojadas em freestall climatizado. Quatro vídeo-câmeras foram instaladas em cada tratamento, nos locais estratégicos do freestall, de modo a capturar, em tempo real o deslocamento dos animais. O experimento teve duração de 28 dias consecutivos do mês de novembro de 2003 , no qual as imagens foram gravadas durante sete dias, no intervalo das 9 às 17 horas. Foram utilizadas 15 vacas em lactação, multíparas, com produção média de $20 \mathrm{~kg}$ de leite dia $^{-1}$. Os tratamentos adotados foram: ausência de ventilação (V0), ventilação (V) e ventilação + nebulização (VN) posicionados na área de descanso dos animais. Os dados referentes à temperatura do bulbo seco (TBS) e umidade relativa do ar (UR) foram mensurados a cada 15 minutos ao longo das 24 horas no interior da instalação e no ambiente externo. A UR mostrou-se mais elevada nos tratamentos V0 $(61,8 \%)$ e $\mathrm{VN}(61,8 \%)$ quando comparada ao tratamento $\mathrm{V}(60,3 \%)$. O THI mostrou-se mais elevado em V $(75,0)$, quando comparado ao tratamento V0 $(74,5)$ e VN $(74,3)$. Os animais submetidos ao tratamento $\mathrm{V}(108,3 \mathrm{~min})$ passaram mais tempo na área de alimentação quando comparados com V0 $(60,7 \mathrm{~min})$ e VN (72,5 min). Os animais do tratamento $\mathrm{V}(19,3 \mathrm{~min})$ permaneceram um período maior na área do bebedouro em relação a V0 (8,3 min) e VN (12,7 min) que não diferiram entre si. Foram observadas diferenças na preferência pelo lado da instalação. A área de descanso apresentava

\footnotetext{
* Parte da tese apresentada pelo primeiro autor à ESALQ/USP no curso de Física do Ambiente Agrícola. Trabalho financiado pela Fundação de Amparo à Pesquisa do Estado de São Paulo (FAPESP)

${ }_{1}$ Pesquisador Científico, Dr., Centro APTA Bovinos de Leite - Instituto de Zootecnia, Nova Odessa-SP, e-mail: matarazzo@iz.sp.gov.br

2 Engenheiro Agrícola, Prof. Dr., Departamento de Engenharia Rural, ESALQ/USP, Piracicaba, SP.

${ }^{3}$ Engenheiro Agrônomo, MSc, Departamento de Engenharia Rural, ESALQ/USP, Piracicaba, SP.

${ }^{4}$ Zootecnista, Prof. Adjunto, Departamento de Tecnologia e Rural e Animal, UESB, Itapetinga, BA

${ }^{5}$ Engenheira Agrônoma, Profa. Dra., Departamento de Construções Rurais, FEAGRI/UNICAMP, Campinas, SP.

${ }^{6}$ Doutor em Engenharia Agrícola, Instituto de Zootecnia, Agencia Paulista de Tecnologia dos Agronegócios, Capta Bovinos Leiteiros, Nova Odessa-SP.

7 Doutora em Engenharia Agrícola, Instituto de Zootecnia, Agencia Paulista de Tecnologia dos Agronegócios, Capta Bovinos Leiteiros, Nova Odessa-SP.
} 
camas adjacentes à área de alimentação e adjacentes ao bebedouro. As vacas dos tratamentos V0 (189,0 min) e V (167,3 min) permaneceram maior tempo nas camas adjacentes à área de alimentação. Por outro lado, as vacas do tratamento VN $(164,0$ min) passaram mais tempo nas camas adjacentes a área do bebedouro em relação aos animais do tratamento $\mathrm{V}$ (26,7 min). Foi constatado maior tempo em pé no corredor próximo ao bebedouro para as vacas do tratamento VN (35,0 min). Esses resultados permitem inferir que as diferenças verificadas na distribuição espacial dos animais no freestall foram sempre associadas à busca pelo recurso de climatização disponível.

Palavras-chave: Resfriamento evaporativo, área de descanso, comportamento, bovinos leiteiros

\section{ABSTRACT}

This research aimed to use electronic monitoring for evaluating behavior patterns of lactating cows in a cooling freestall. Four video cameras were strategically installed in each treatment for capturing in real time images of animal displacements and activities, as intake, rumination and resting. The trial was carried out during 28 consecutive days of November 2003, in which images were daily recorded from 9 a.m. to 5 p.m. during 7 days. Fifteen multiparous lactating cows producing $20 \mathrm{~kg}$ of milk per day were used. The tested treatments were: non-fan (FO), fan (F) and fan plus misting (FM) in direction to animal bedding. Dry bulb temperature (DBT) and air relative humidity $(\mathrm{RH})$ inside and outside of facility were measured every 15 minutes during 24 hours. The $\mathrm{RH}$ was higher in non-fan (61.8\%) and fan plus misting (61.8\%) treatments than in fan $(60.3 \%)$ treatment. Also, the temperature and humidity index was higher in fan (75.0) treatment than in nonfan (74.5) and fan plus misting (74.3) treatments. In the treatment with fan, animals spent more time in the feeding area $(108.3 \mathrm{~min})$ than those of non-fan $(60.7 \mathrm{~min})$ and fan plus misting (72.5 min) treatments. Fan treatment cows spent more time (19.3 min) in waterers in relation to non-fan $(8.3 \mathrm{~min})$. Different patterns were observed in the cow's preference relative to resting area. The resting areas include the bedding adjacent to feeding area and bedding adjacent to waterers. Cows of non-fan (189.0 min) and fan (167.3 min) spent more time lying in the bedding adjacent to feeding area. However, the cows of treatment fan plus misting ( 164.0 min of total) spent more time lying in the bedding adjacent to waterers in relation fan $(26.7 \mathrm{~min})$. The cows of fan plus misting treatment also spent more time (35.0 min) standing in passage area (drinkers) than of non-fan and fan (10.0 min). In the current experiment, the results indicate difference in freestall occupancy. The cow's preference was always related to the availability of cooling system.

Keywords: Evaporative cooling, resting area, behaviour, dairy cows.

\section{INTRODUÇÃO}

A resposta de um ser vivo ao ambiente no qual está inserido é geralmente adaptativa, ou seja, as possibilidades de sobrevivência da espécie são aumentadas se o animal consegue regular sua resposta de modo apropriado às mudanças das condições ambientais. Geralmente, essas reações são movimentos da totalidade ou parte de seu corpo ou mesmo da inatividade, a qual se mostra uma reação significativa e diretamente relacionada à mudança no ambiente (BOWLER, 1993).

Determinada situação ambiental, normalmente, estimula mais que uma 
resposta comportamental, mas o animal aprende a empregar aquela que mais se mostra eficiente (Curtis, 1981). De acordo com Pires et al. (1998), embora existam poucas referências sobre a extensão das mudanças comportamentais em condições de estresse térmico, fica claro que as alterações dos padrões usuais de ingestão de água e alimentos, postura e movimentação são importantes mecanismos adaptativos para reduzir o efeito do calor (PIRES et al., 2000).

O sistema de produção freestall tem sido planejado para maximizar a densidade de animais e a eficiência de manejo, mas nem sempre proporciona condições de conforto para as vacas em lactação (OVERTON et al., 2002). Trabalhos recentes relataram que o uso do freestall pode ser um indicador da preferência da vaca, e essa preferência pode ser interpretada como medida do seu conforto (WAGNERSTORCH et al., 2003).

O controle eficiente do ambiente pode empregar sistemas naturais e artificiais. Inúmeros métodos têm sido desenvolvidos para modificar o ambiente visando a amenizar o efeito do estresse térmico. A movimentação do ar, o umedecimento da superfície do animal, o resfriamento evaporativo do ar e o uso de sombras para minimizar o efeito da radiação solar, são artifícios empregados para potencializar a dissipação de calor (WEST, 2003).

Tem se constatado que a instalação dos sistemas de aspersão ou nebulização na linha de alimentação leva a vaca a permanecer durante longos períodos nesses locais, a fim de se beneficiarem da remoção do calor corporal por meio da evaporação da água na pele. Por outro lado, esses resultados indicaram presença do estresse térmico, considerando que ocorreu aumento no tempo em que os animais ficaram em pé (CALEGARI et al., 2003).

Dentre os padrões fixos de comportamento, o de deitar é considerado de grande importância para as vacas leiteiras. A privação do descanso pode induzir à frustração que se manifesta por comportamentos estereotipados. Outras conseqüências incluem lesões traumáticas e outros danos físicos, resultando em problemas sanitários e baixo desempenho produtivo (KROHN \& MUNKSGAARD, 1993). Sendo assim, CALEGARI et al. (2003) sugeriram também, a possibilidade de estender o sistema de resfriamento evaporativo nas demais instalações, como por exemplo, na área de descanso de modo a evitar a aglomeração dos animais na linha de alimentação.

Em verdade, mediante diversas formas de comportamento, as vacas buscam seu bem-estar. Cabe ao homem auxiliar os animais, provendo-Ihes um ambiente adequado, que seja ao mesmo tempo confortável e produtivo (BACCARI JUNIOR, 2001). Desta forma, o trabalho teve como objetivo empregar o monitoramento eletrônico para avaliar as respostas comportamentais de vacas em lactação alojadas em freestall climatizado.

\section{MATERIAL E MÉTODOS}

O experimento foi conduzido com rebanho comercial de gado Holandês, localizado no município de São Pedro, SP, a altitude de $580 \mathrm{~m}$ e coordenadas $22^{\circ} 33^{\prime} 02^{\prime \prime}$ de latitude Sul e $47^{\circ} 38^{\prime} 05^{\prime \prime}$ de longitude Oeste. O clima da região é do tipo Cwa da classificação Köppen, ou seja, quente e úmido com estação chuvosa no verão e seco no inverno.

A instalação apresentava as seguintes características construtivas: $80 \mathrm{~m}$ de comprimento e $28 \mathrm{~m}$ de largura, laterais abertas, orientação leste-oeste, corredor central de $3,0 \mathrm{~m}$, pé-direito central de $9 \mathrm{~m}$, telhado de duas águas com cobertura de telha de barro e sistema de lanternim vazado. O piso era de concreto e apresentava ranhuras de $0,5 \mathrm{~cm}$ espaçadas $7 \mathrm{~cm}$ entre si. A linha de alimentação tinha a extensão de todo galpão. Os bebedouros eram de alvenaria, localizados estrategicamente nas laterais do galpão, com dimensões de 2,8 m de comprimento, $0,8 \mathrm{~m}$ de largura, $0,31 \mathrm{~m}$ de profundidade e capacidade de $0,8 \mathrm{~m}^{3}$ de água. 
Foram utilizadas 15 vacas Holandesas, pluríparas, em lactação, com peso médio de $600 \mathrm{~kg}$ e produção média de $20 \mathrm{~kg}$ de leite por dia. Os animais permaneceram durante todo dia com livre acesso às camas e demais áreas do abrigo, mas sem acesso as pastagens. O manejo dos animais durante a fase experimental consistiu de dois horários de alimentação (10 e 16 horas) e três horários de ordenha (1,9 e 17 horas).

O período experimental teve a duração de 28 dias consecutivos no mês de novembro de 2003 , dos quais sete dias foram destinados à adaptação dos animais ao tratamento e manejo empregado, e 21 dias para registro dos dados referentes às variáveis ambientais, produtivas e fisiológicas das vacas em lactação.

O freestall foi equipado com sistemas de aspersão, o qual foi montado em toda extensão do da linha de alimentação, a altura de $2,5 \mathrm{~m}$, com espaçamento entre bicos de $1 \mathrm{~m}$ e constituído por tubo PVC. Esse sistema era acionado por uma bomba com motor trifásico cujo consumo de energia era equivalente a $1,48 \mathrm{~kW} \mathrm{~h}^{-1}$. A vazão de água na linha de aspersão foi de $30 \mathrm{~L} \mathrm{hora}^{-1}$ e a intermitência foi de $12 \mathrm{~min}$. No SRAE utilizaram-se ventiladores com diâmetro de $0,9 \mathrm{~m}$, espaçados a cada $11 \mathrm{~m}$, equipados com motor de $1 / 4 \mathrm{CV}$, vazão de $300 \mathrm{~m}^{3}$ hora1, 495 RPM, e capacidade de produzir movimentação de ar de até $2,5 \mathrm{~m} \mathrm{~s}^{-1}$. $\mathrm{Na}$ área de alimentação esses ventiladores foram fixados a altura de $2,5 \mathrm{~m}$ e na área de descanso a $2 \mathrm{~m}$.

Os tratamentos foram aplicados aleatoriamente na área de descanso dos animais da seguinte maneira: Tratamento 1: sem ventilação (V0); Tratamento 2: ventilação forçada (V); Tratamento 3: ventilação forçada adicionada de linha de nebulização (VN). O sistema de nebulização foi montado a altura de 3,7 m acima da área de descanso dos animais e era constituída por tubo PVC, com espaçamento entre bicos de $1 \mathrm{~m}$. A vazão de água na linha de nebulização foi de $3 \mathrm{~L}_{\text {hora }}^{-1}$ e a intermitência foi a mesma do sistema de aspersão (12 min) uma vez que eram alimentados pela mesma bomba. Esse sistema era alimentado por uma bomba com motor trifásico cujo consumo de energia era equivalente a $0,74 \mathrm{~kW}_{\text {hora }}{ }^{-1}$. O SRAE e os ventiladores foram acionados por meio de termostato toda a vez que a temperatura no interior do freestall atingia $25^{\circ} \mathrm{C}$.

As variáveis registradas no interior das instalações e no ambiente externo foram a temperatura do bulbo seco (TBS) e umidade relativa do ar (UR\%). As leituras foram realizadas a cada 15 minutos e então estimada a média horária. A aquisição dos dados foi realizada por sensores acoplados a um sistema eletrônico ( $\mathrm{HOBO}$ 'H8). A estação autmomática foi fixada a altura de 2,5 $\mathrm{m}$ do piso, a fim de protegê-los dos animais. A partir dos valores encontrados para as variáveis meteorológicas, foram calculados o índice de conforto térmico THI (THOM, 1959) e a entalpia. Para cálculo da entalpia a equação foi descrita por VILLA NOVA (1999), citado por FURLAN (2001).

Dentro da seção do freestall estudada para cada tratamento, foi realizada uma subdivisão, correspondente a $12 \mathrm{~m}$ de comprimento por $12 \mathrm{~m}$ de largura. Este espaço físico dispunha de 14 camas dispostas frente a frente, permitindo que todas as vacas tivessem a mesma oportunidade de escolher o lado desejado para deitar. A restrição da área foi necessária, em função da limitação do alcance das câmeras empregadas.

Quatro vídeo-câmeras coloridas, com lente de 2,45 $\mathrm{mm}$ foram alocadas estrategicamente no freestall, à altura de 2,75 m acima do piso de forma a permitir a visualização completa da área em estudo. As câmeras foram acopladas ao microcomputador central que era equipado com placa de captura de imagens, sendo estas gerenciadas em tempo real pelo software Topway'. As imagens foram registradas diariamente, durante sete dias, no intervalo das 09h:00min às 17h:00min.

Para quantificar os padrões comportamentais, os vídeos foram revistos, e a cada intervalo de dez minutos era anotado o comportamento animal no etograma previamente estabelecido. A partir 
destes dados, determinou-se o tempo em minutos e em porcentagem em que os animais exerceram cada atividade. Os horários destinados à ordenha dos animais foram desconsiderados da análise. Os animais foram monitorados quanto a sua localização dentro da instalação: presença na área de alimentação, presença na área do bebedouro, presença na cama próxima à área de alimentação, presença na cama próxima à área do bebedouro, presença no corredor próximo á área de alimentação e presença no corredor próximo à área do bebedouro (Figura 1).

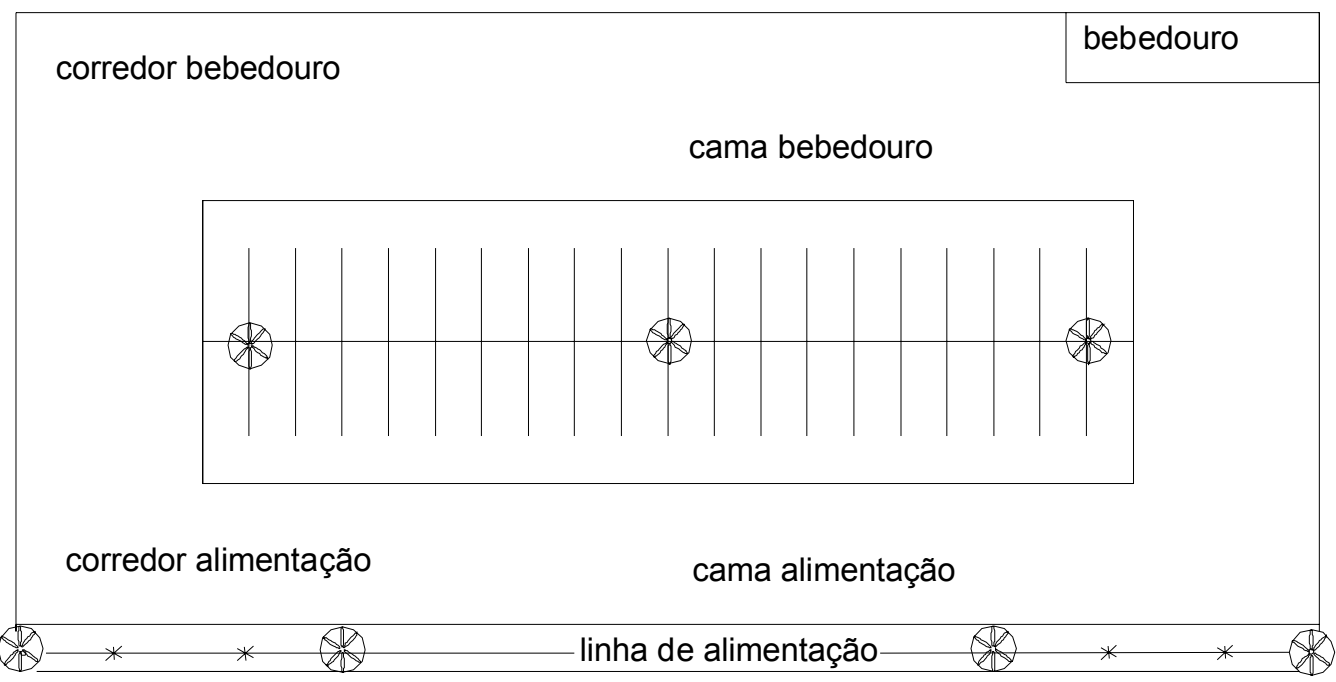

ventilador

aspersor

Figura 1. Representação esquemática dos locais avaliados.

Para a análise estatística das variáveis ambientais (TBS, UR, THI e H) adotou-se um delineamento experimental em blocos casualizados, com os dias sendo usados como repetições. Para o comportamento animal, adotou-se o delineamento inteiramente casualizado, utilizando os animais como repetições. Empregou-se o SAS/LAB para verificar as pressuposições da análise de variância (ANOVA) para cada comportamento. Quando as pressuposições da ANOVA foram satisfeitas a comparação de médias foi realizada pelo Teste de Tukey ao nível de significância de 5\%. Quando as pressuposições da ANOVA não foram atendidas, os tratamentos foram comparados utilizando-se a macro Kruskall.mac do Minitab, com a aplicação do teste não-paramétrico de Kruskal-Wallis e comparações múltiplas das medianas pelo teste de Dunn (GIBBONS, 1976; NOETHER, 1991).

\section{RESULTADOS E DISCUSSÃO}

Os valores médios encontrados para a temperatura de bulbo seco (TBS), umidade relativa (UR) e para o índice de temperatura e umidade (ITU) durante o período experimental são apresentados na Tabela 1.

Tabela 1. Valores médios e erros padrão da média das variáveis meteorológicas e índices de conforto térmico registrados no intervalo horário das 9 às 17 horas durante o período experimental.

\begin{tabular}{lccc}
\hline Variáveis meteorológicas & \multicolumn{3}{c}{ Tratamentos } \\
& V0 & $\mathrm{V}$ & $\mathrm{VN}$ \\
\hline Temperatura de bulbo seco, ${ }^{\circ} \mathrm{C}$ & $26,6 \pm 0,11 \mathrm{a}$ & $26,8 \pm 0,11 \mathrm{a}$ & $26,6 \pm 0,11 \mathrm{a}$ \\
Umidade relativa, \% & $61,8 \pm 0,34 \mathrm{a}$ & $60,3 \pm 0,34 \mathrm{~b}$ & $61,8 \pm 0,34 \mathrm{a}$ \\
Índice de temperatura e umidade & $74,5 \pm 0,14 \mathrm{a}$ & $75,0 \pm 0,14 \mathrm{~b}$ & $74,3 \pm 0,14 \mathrm{a}$ \\
\hline
\end{tabular}

a, b Médias seguidas por letras minúsculas distintas nas linhas diferem entre si pelo Teste $t$ de Student $(\mathrm{P}<0,05)$. 
Não foram observadas diferenças $(P>0,05)$ entre os tratamentos para a TBS. Os valores médios encontrados foram 26,6 ; 26,8 e $26,6^{\circ} \mathrm{C}$, respectivamente, para os tratamentos $\mathrm{V} 0, \mathrm{~V}$ e $\mathrm{VN}$. Os valores relatados na literatura indicam grande variação na temperatura crítica superior para as vacas em lactação. BENJAMIM et al. (1985) apontaram valores entre 25 e $26^{\circ} \mathrm{C}$, por outro lado, HUBER (1990) citou a faixa de 4 a $26^{\circ} \mathrm{C}$ como de conforto térmico para as vacas em lactação. Nas condições do presente experimento, verificou-se que os valores da TBS estiveram dentro do padrão de termoneutralidade.

Verificaram-se diferenças $(P<0,05)$ entre os tratamentos na UR. Esta variável mostrou-se mais elevada nos tratamentos V0 e VN, quando comparada ao tratamento $\mathrm{V}(60,3 \%)$. Entretanto, os tratamentos V0 e VN não diferiram entre si apresentando valores correspondentes a $61,8 \%$ de UR. Os tratamentos avaliados não resultaram em umidade excessiva, ou seja, maior que $70 \%$ no interior da instalação. Temperatura ambiente e umidade relativa elevada poderiam limitar o desempenho das vacas confinadas, pois diminuem sua habilidade em dissipar calor. Isso ocorre porque, em condições de umidade elevada, o ar saturado irá inibir a evaporação da água pela pele e sistema respiratório, proporcionando um ambiente ainda mais estressante para o animal (SOTA, 1996).

Foram encontradas diferenças $(P<0,05)$ entre os tratamentos para o ITU. Este índice mostrou-se mais elevado no tratamento $\mathrm{V}(75,0)$, quando comparado ao tratamento V0 $(74,5)$ e VN $(74,3)$, sendo que esses últimos não diferiram entre si. De acordo com Hahn (1985), um ITU igual ou menor a 70 expressa uma condição normal; um valor entre 71 e 78 é crítico; entre 79 e 83 a situação é de perigo e, acima de 83, uma situação de emergência está presente. Nas condições do presente experimento, verificou-se que o ITU permaneceu na faixa considerada crítica. Sendo assim, os equipamentos de climatização não possibilitaram o condicionamento ambiental relativamente eficiente nas horas mais quentes do dia.

O período médio diário em que os animais foram monitorados pelas câmeras de vídeo foi de 6 horas e 30 minutos (376 $\mathrm{min}$ ), sendo que os horários destinados à ordenha dos animais foram desconsiderados da análise. A Tabela 2 e Figura 2 apresentam o tempo médio, em minutos, que os animais permaneceram na área de alimentação, no bebedouro, nas camas próximas à linha de alimentação, nas camas próximas à área do bebedouro, no corredor próximo à linha de alimentação e no corredor próximo à área do bebedouro.

Os valores obtidos para o tempo de permanência na área de alimentação indicaram diferenças $(P<0,05)$ entre os tratamentos. Os animais submetidos ao tratamento $\mathrm{V}$ passaram mais tempo neste local, sendo equivalente a 108,3 min $(28,8 \%$ do tempo total) quando comparados com V0 que ficaram $60,7 \mathrm{~min}$ (16,3\% do tempo total) e VN que permaneceram 72,5 min $(18,8 \%$ do tempo total). O tempo de permanência dos animais nos tratamentos V0 e VN não diferiram entre si.

Entretanto, o maior tempo despendido pelos animais do tratamento $\mathrm{V}$ na linha de alimentação não refletiu em aumento no consumo médio de matéria seca, uma vez que $\mathrm{V}(21,3 \mathrm{~kg}$ MS dia-1) e V0 (20,5 kg MS dia-1) não diferiram entre si (Matarazzo, et al. 2005). Possivelmente, esses animais permaneceram por um período maior junto ao cocho, local no qual se apresentava o sistema de aspersão, com objetivo de maximizar o resfriamento corporal, e não necessariamente em busca de alimento.

Este trabalho corrobora os resultados encontrados por CALEGARI et al. (2003). Os autores verificaram que os animais do tratamento com nebulização apresentaram maior tempo despendido em pé, na área de alimentação, sendo esta uma situação indicativa de estresse térmico, já que as vacas procuraram ficar com maior área de superfície corporal exposta, a fim de facilitar as trocas de calor. 
Tabela 2. Tempo médio (minutos) em que os animais permaneceram em cada local dentro da instalação nos tratamentos avaliados.

\begin{tabular}{lccr}
\hline Localização & V0 & $\mathrm{V}$ & $\mathrm{VN}$ \\
\hline Área de alimentação & $60,7 \pm 24,3 \mathrm{~b}$ & $108,3 \pm 15,7 \mathrm{a}$ & $72,5 \pm 17,7 \mathrm{~b}$ \\
Área do bebedouro & $8,3 \pm 4,9 \mathrm{~b}$ & $19,3 \pm 5,2 \mathrm{a}$ & $12,7 \pm 4,2 \mathrm{ab}$ \\
Cama (área de alimentação) & $189,0 \pm 52,2 \mathrm{a}$ & $167,3 \pm 18,0 \mathrm{a}$ & $82,1 \pm 39,8 \mathrm{~b}$ \\
Cama (área do bebedouro) & $51,7 \pm 33,4 \mathrm{AB}$ & $26,7 \pm 12,8 \mathrm{~B}$ & $164,0 \pm 83,2 \mathrm{~A}$ \\
Corredor (área de alimentação) & $53,3 \pm 33,9 \mathrm{~A}$ & $45,0 \pm 23,3 \mathrm{~A}$ & $20,0 \pm 26,3 \mathrm{~A}$ \\
Corredor (área do bebedouro) & $10,0 \pm 5,6 \mathrm{~B}$ & $10,0 \pm 3,9 \mathrm{~B}$ & $35,0 \pm 27,1 \mathrm{~A}$ \\
\hline
\end{tabular}

a,b Médias seguidas por letras minúsculas distintas nas linhas diferem pelo Teste de Tukey $(P<0,05)$

$A, B$ Médianas seguidas por letras maiúsculas distintas nas linhas diferem pelo Teste de Kruskal-Wallis $(P<0,05)$

Com relação ao tempo de permanência na área do bebedouro, verificaram-se diferenças $(P<0,05)$ entre os tratamentos. Os animais mantidos no tratamento $\mathrm{V}$ permaneceram um período maior neste local, sendo este correspondente a $19,3 \mathrm{~min}$ (5,1\% do tempo total) quando comparados com $\mathrm{V} 0$ que passaram $8,3 \mathrm{~min}(2,2 \%$ do tempo total). Os animais submetidos a VN ficaram 12,7 min (3,3\% do tempo total), sendo que o tempo de permanência dos animais no tratamento VN não diferiu entre $\mathrm{V} 0 \mathrm{e} \mathrm{V}$. O consumo de água por vaca em lactação depende de vários fatores: estado fisiológico, produção de leite, peso corporal, raça, consumo de matéria seca, composição da dieta, ambiente, clima e qualidade da água. Durante os meses mais quentes, as vacas sofrem estresse pelo calor e pela elevação da umidade relativa do ar, aumentando o consumo de água, com elevação na excreção de urina e alterando a composição dos dejetos (CAMPOS, 2003).

Quando avaliado o tempo em que os animais permaneceram na área de descanso, constataram-se diferenças $(P<0,05)$ entre os tratamentos. Para o tempo de permanência na cama próxima à linha de alimentação, os resultados encontrados foram $189,0 \mathrm{~min}(50,7 \%$ do tempo total), $167,3 \mathrm{~min}(44,4 \%$ do tempo total) respectivamente, para os tratamentos $\mathrm{V} 0$, V. O tempo despendido pelos animais em VN foi $82,1 \mathrm{~min}(21,3 \%)$, sendo este último, diferente dos demais. Com relação à permanência na cama oposta à linha de alimentação, os valores encontrados foram
$51,7 \mathrm{~min}$ (13,9\% do tempo total), $26,7 \mathrm{~min}$ (7,1\% do tempo total) e 164,0 min $(42,5 \%$ do tempo total), respectivamente, para os tratamentos $\mathrm{V} 0, \mathrm{~V}$ e $\mathrm{VN}$, sendo esses dois últimos diferentes entre si.

As vacas tiveram livre acesso aos lados do freestall, sendo assim, todas dispunham da mesma oportunidade de escolher o local desejado para se deitar. A maior procura pelas camas próximas à linha de alimentação verificada nos animais submetidos ao tratamento $\mathrm{V} 0$ e $\mathrm{V}$ pode estar associada à presença de climatização (aspersão/ventilação) na linha de alimentação. Provavelmente, as vacas sentiram-se atraídas para este lado da instalação, dado ao efeito associativo da aspersão/ventilação no cocho, o qual pode ter promovido alteração no microclima dessa área do freestall, proporcionado, assim, sensação de conforto aos animais.

GAWORSKI et al (2003) também constataram um número maior de visitas para as camas próximas à linha de alimentação, sendo que as camas localizadas no centro do freestall foram preferidas em relação às camas distribuídas na periferia. Esses resultados indicaram que as camas afastadas da linha de alimentação ou na periferia são menos desejáveis para as vacas, talvez porque precisem caminhar mais ou por encontrarem obstáculos sociais (vacas dominantes) em seu caminho.

Por outro lado, verificou-se maior procura pelas camas próximas ao bebedouro nos animais mantidos em VN. Cabe ressaltar que neste local, o sistema de nebulização instalado para compor o 
tratamento encontrava-se, na realidade, no corredor próximo à área do bebedouro e não dirigido à área de descanso, como era esperado. Estes dados confirmaram também a preferência dos animais deste mesmo tratamento a permanecerem por um período maior neste lado do freestall.

Os valores obtidos para o tempo de permanência no corredor junto à área de alimentação não foram diferentes $(P>0,05)$ entre os tratamentos, sendo os valores correspondentes a $53,3 \mathrm{~min}(14,3 \%$ do tempo total), $45 \mathrm{~min}(11,9 \%$ do tempo total) e $20 \min (5,2 \%$ do tempo total $)$, respectivamente, para os tratamentos $\mathrm{V} 0$, $\mathrm{V}$ e VN. Por outro lado, quando avaliados os resultados encontrados para o tempo de permanência no corredor próximo à área do bebedouro, constataram-se diferenças $(P<0,05)$ entre os tratamentos, sendo que os animais mantidos em $\mathrm{V} 0$ e $\mathrm{V}$ ficaram 10 $\min (2,7 \%$ do tempo total) neste local, período significativamente menor, quando comparados com VN que passaram $35 \mathrm{~min}$ ( $9,1 \%$ do tempo total).

Além do tratamento VN apresentar o sistema de nebulização deslocado para o corredor, fazendo com que os animais permanecessem mais tempo nesse local, a face lateral do galpão permanecia exposta ao ar livre, sem qualquer tipo de construção ou obstáculos no entorno. Tal fato, provavelmente, favoreceu a dissipação de calor dos animais com o meio, estimulandoos a permanecer por mais tempo neste local.

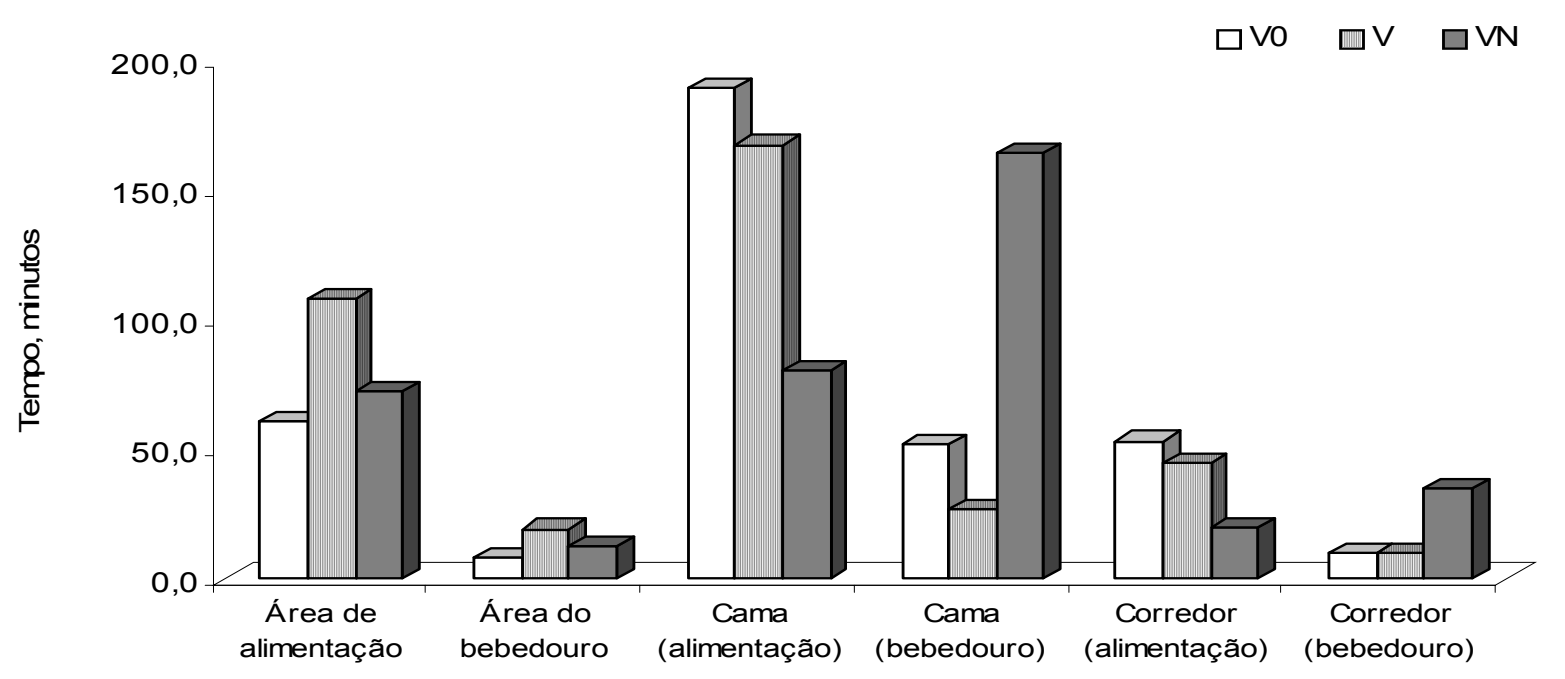

Figura 2. Tempo (minutos) em que os animais permaneceram nos locais dentro da instalação.

\section{CONCLUSÕES}

Conforme os resultados verificados nas condições do presente experimento, pode-se concluir que a climatização da área de descanso em instalações do tipo freestall não proporcionou melhorias nas propriedades psicrométricas do ar e que as diferenças verificadas na distribuição espacial dos animais no freestall foram sempre associadas à busca pelo recurso de climatização disponível. 


\section{REFERENCIAS BIBLIOGRÁFICAS}

BACCARI JR., F. Manejo ambiental da vaca leiteira em climas quentes. Londrina: UEL, 2001. 142p

BOWLER, P. J. The environmental sciences. New York: W.W Norton and Company, 1993. 315p.

CALEGARI, F.; CALAMARI, L.; FARZZI, E. Effects of ventilation and misting on behavior of dairy cattle in the hot season in south Italy. In: INTERNATIONAL DAIRY HOUSING CONFERENCE, 5. Texas, 2003. Proceedings. Texas: American Society of Agricultural Engineers, 2003. p. 303-311.

CAMPOS A. T. A importância da água para o gado de leite. Embrapa Gado de Leite. Disponível em: <http://www.sbz.org.br>. Acesso em 20 de mar. de 2003.

CURTIS, S. E. Environment management in animal agriculture. Illinois: Animal Environment Services, 1981. 430 p.

FURLAN, R.A. Avaliação da nebulização e abertura de cortinas na redução da temperatura do ar em ambiente protegido. Piracicaba, 2001. 146 p. Tese (Doutorado) - Escola Superior de Agricultura "Luiz de Queiroz", Universidade de São Paulo.

GAWORSKI, M. A.; TUCKER, C. B.; WEARY, D. M. Effects of stall design on dairy cattle behaviour. (Compact disc). In: INTERNATIONAL DAIRY HOUSING CONFERENCE, 5., Fort Worth, 2003. Proceedings. Fort Worth: American Society of Agricultural Engineers, 2003.

GIBBONS, JEAN D. Nonparametric methods for quantitativea analysis. New York: Holt, 1976. 254p.

KROHN ,C.C.; MUNKSGAARD, L. Behaviour of cows kept in extensive (loose housing/pasture) or intensive (tiestall) environment. Applied Animal Behaviour Science, v.37, p1-6, 1993.
MATARAZZO, S. V.; PERISSINOTTO, M.; SILVA, I. J. O. ; MOURA, D. J. FERNANDES,S.A.A. Thermal conditioned in resting area of freestall facilities and its consequences on productive and physiological responses in dairy cows. Paper 054020. American Society of Agricultural Engineers, 2005.

NOETHER, G. E. Introduction to statistics: the nonparametric way. New York: SpringerVerlag, 1991.

OVERTON, M. W.; SISCHO, W.M.; TEMPLE, G. D. Using time-lapse video photography to assess dairy cattle lying behavior in freestall barn. Journal of Dairy Science, Savoy, IL.v.85, p.2407-2413, 2002.

PIRES, M. F. A.; VILELA, D.; VERNEQUE, R. S.; TEODORO, R. L Reflexos do estresse térmico no comportamento das vacas em lactação. In: SIMPÓSIO BRASILEIRO DE AMBIÊNCIA NA PRODUÇÃO DE LEITE, 1., Piracicaba, 1998. Anais... Piracicaba: FEALQ, 1998. p.68-102.

PIRES, M. F. A.; TEODORO, R. L.; CAMPOS, A. T. Efeitos do estresse térmico sobre a produção de bovinos. In: CONGRESSO NORDESTINO DE PRODUÇÃO ANIMAL, 2. Teresina, 2000. Anais... Teresina: SNPA, p.87-104, 2000.

THOM, E. C. The discomfort index. Weatherwise, Washington, v.12, p.57-59, 1959.

WAGNER-STORCH, A. M.; PALMER, R. W.; KAMMEL, D. W. Factors affecting stall use for different freestall bases. Journal of Dairy Science, Savoy, IL, v.86, p.2253-2266, 2003.

WEST, J. W. Effects of heat-stress on production in dairy cattle. Journal of Dairy Science, Savoy, IL, v.86, p.2131-2144, 2003. 